\title{
Prediction method of landslide disaster in southern China based on multi attribute group decision making
}

\author{
ZHANG Si-yuan $^{1, *}$, REN Wei-zhong ${ }^{1}$, ZHANG Xin-xin $^{1}$, LIU Hai-kang $^{1}$ \\ 1.State Key Laboratory of Geomechanics and Geotechnical Engineering, Institute of Rock and Soil \\ Mechanics, Chinese Academy of Sciences, Wuhan 430071, China \\ *.Email: zsy19881107@163.com
}

Keywords: landslide; prediction model; geological analysis; decision-making model

\begin{abstract}
According to prediction of landslide based on grey vector machine model, the landslide is affected by multiple factors such as soil layer structure and these factors show complicated non-linear relation with landslide, while it is hard for the traditional model to reflect the non-linear complicated relation. The prediction model for law of landslide established by grey model and neural network model can better predict the complicated surface subsidence and can effectively improve the predicted precision compared with the single model. Not only the reliability of prediction system is improved, but also the cost of the whole system is reduced.
\end{abstract}

\section{Introduction}

Due to reasons such as landform and human engineering activities, the landslide accidents frequently occur in some regions in China, and there are more than 140 large-scale landslides developed and more than 2212 relatively large landslides. In case of landslide, not only the casualties and property loss on landslide mass can be caused, but also the debris flow can endanger the housing, traffic and personnel safety in a certain scope; so with regard to the problems such as difficulties in prevention, rescue and governance and serious damages of landslide, people are mainly concerned about how to promptly and effectively monitor the state of mountains, to discover the abnormal state in advance, to promptly give a alarm, etc. Some landslides instantly occur under the action of inducing factors, such as occurrence of earthquake, rainstorm, tsunami, storm tide, etc. and unreasonable human activities of excavation, explosion, etc. which can result in a large number of landslides. Some landslides occur slightly later than the action time of inducing factors, such as rainfall, snow melt, tsunami, storm tide, and human activities. The laggard law is most evident in rainfall-induced landslide which tends to occur after rainstorm, heavy rain and long-time continuous rainfall and the length of laggard time is related to lithology of landslide mass, structure and the amount of rainfall. In general, the looser the landslide mass is, the more developed the crack is and the heavier the rainfall is, then the shorter the laggard time is. In addition, the landslides caused by artificial excavation of slope toe, piling loading and water storage and discharge also fall within the category. The length of laggard time for landslide induced by human activities is related to strength of human activities and original stability of landslide mass. The greater the strength of human activities is and the lower the stability of landslide mass is, the shorter the laggard time is. The bank slope regions of river, lake (reservoir), sea and ditch, canyon with high altitude difference in landform, and the side slope regions of mountain area, railway, highway, and engineering buildings provide favorable landform conditions for landslide; in geologic tectonic zones such as fault zone and seismic zone, in general, when the seismic intensity is greater and the gradient is greater than 25 degrees, the landslide is extremely liable to occur during earthquake; the broken rock mass and developed crack in fault zone are favorable for landslide; rock and soil distribution zones liable for landslide. For example, rock and soil such as loose cover layer, loess, mud rock, shale, coal measure strata, tuff, schist, killas and phyllite provide favorable material basis for landslide; regions with frequent rainstorm or abnormal strong rainfall. In these regions, the abnormal rainfall provides favorable inducing factors for landslide. The overlapped region of the above zones forms the 
densely developed region of landslide. The regions from Taihang Mountains to Qinling Mountains and through western Hubei, Sichuan, Yunnan and eastern Tibet belong to the typical area with landslide occurring more densely and serious harm.

\section{Prediction model for landslide of grey neural network}

\section{prediction model}

The historical data of problem to be predicted is used for grey prediction, the grey model is adopted for learning to construct the corresponding prediction model and then predict the value for future time by the model.

$G M(m, n)$ indicates the grey model of a $m$ order and $n$ variables. Suppose that the original data sequence of landslide is $x^{(0)}$ and obtain the new data sequence $x^{(1)}$ after one accumulation, so $x^{(1)}$ satisfies the first order differential equation:

$$
\frac{d x^{(1)}}{d t}+\alpha x^{(1)}=u
$$

Where, $\alpha$ and $u$ are the parameters to be estimated of differential equation and they conform to the following relation equation.

$$
x^{\wedge(1)}(i+1)=\left(x^{(1)}-\frac{u}{\alpha}\right) e^{-\alpha i}+\frac{u}{\alpha}
$$

Then solve the parameters of $\alpha$ and $u$ to be estimated through least square method and the equation is as follows:

$$
\widehat{A}=\left[\begin{array}{l}
\alpha \\
u
\end{array}\right]=\left(B^{T} B\right)^{-1} B^{T} Y_{N}
$$

Where

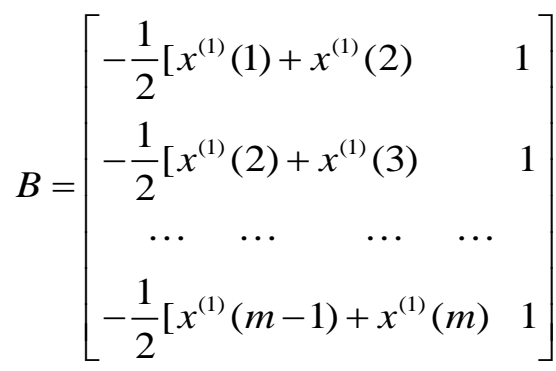

$$
\begin{aligned}
& Y_{N}=\left(x^{(0)}(2), x^{(0)}(3), \cdots, x^{(0)}(m)\right)^{T}
\end{aligned}
$$

The values for the parameters of $\alpha$ and $u$ are obtained after Equation (4) and Equation (5), then put the values in Equation (1) and solve the differential equation to obtain the prediction model of $\operatorname{GM}(1,1)$ described as follows:

$$
\widehat{X}^{(1)}(t+1)=\left(x^{(0)}(1)-\frac{u}{\alpha}\right) e^{-\alpha t}+\frac{u}{\alpha}
$$

\section{Prediction model of BP neural network}

BP neural network is a multilayer feedforward neural network and includes input layer, hidden layer and output layer among which the hidden layer can be multilayer with different layers interlinked but the same layer not linked. The hidden layer can be one layer or multiple layers as shown in Fig.1. 


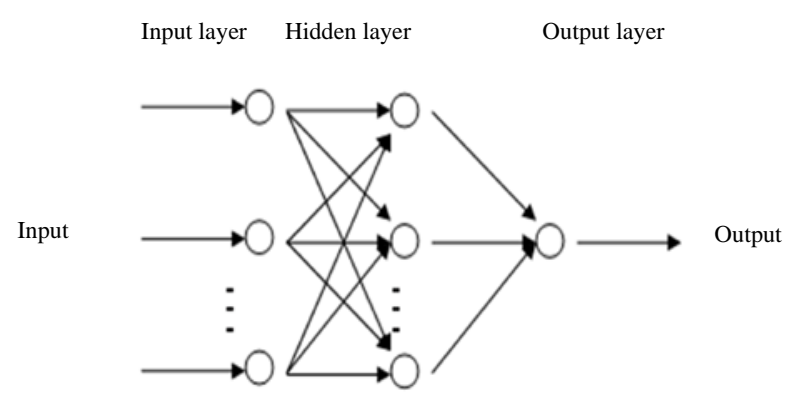

Fig.1 Structure Chart for BP Neural Network

S-type transformation function is generally adopted for transfer function of fundamental processing unit of BP network with simple network structure and strong operability, it can simulate the arbitrary non-linear input and output relations and is of good generalization ability and non-linear approaching ability to simulate landslide. When the BP neural network is determined, the landslide data sequence can be adopted to train the network, i.e. learn and train the weight and threshold of network to realize the given input and output mapping relation.

Suppose that the input of BP neural network is $X=\left(x_{1}, x_{2}, \cdots, x_{n}\right)^{T}$; the neuronal introduction threshold of hidden layer is $x_{0}=-1$ and the input vector of hidden layer is $\mathrm{O}=\left(o_{1}, o_{2}, \cdots, o_{j}\right)^{T}$; the output vector and expected output vector are respectively $Y=\left(y_{1}, y_{2}, \cdots, y_{m}\right)^{T}$ and $T=\left(t_{1}, t_{2}, \cdots, t_{m}\right)^{T}$; $w_{i j}$ and $v_{k j}$ are weight values and Sigmoid function is the excitation function of BP neural network:

$$
f(x)=\frac{1}{1+e^{-x}}
$$

Make ${ }^{o_{i}}$ the actual output of neural network node $i$ and the $j$ neuronal input of hidden layer is shown in Equation (8):

$$
n e t_{j}=\sum_{i=0}^{n} w_{j i} x_{i}
$$

The $j$ neuronal output of hidden layer is shown in Equation (9):

It can be inferred that the $k$ neuronal input of output layer is:

$$
o_{j}=f\left(\text { net }_{j}\right)=\frac{1}{1+e^{\sum_{i=0}^{n} w_{j i} x_{i}}}
$$

$$
n e t_{k}=\sum_{j=0}^{l} v_{k j} o_{j}
$$

Finally, it can be inferred that the $k$ neuronal output of output layer is shown in the following equation:

\section{Grey neural network prediction of landslide}

$$
y_{m}=f\left(\text { net }_{k}\right)=\frac{1}{1+e^{\sum_{j=0}^{l} v_{k j} o_{j}}}
$$

The sample data amount required by the grey prediction model is less, the computation is simple and the prediction precision is relatively high, but the prediction precision for complicated non-linear relation is not high and the non-linear function cannot be approached. BP neural network is of the knowledge-processing thought, learning and memorizing abilities, high non-linear processing ability and good generalization ability. To improve the prediction precision for landslide, the combination of grey model and BP neural network model can not only fill the gap of low solution precision for non-linear function by grey model, but also give play to the advantages of both and precisely reflect the change rule and trend of input and output. 


\section{Example analysis}

\section{Data source of landslide}

To verify the performance of grey neural network in landslide prediction, 21 samples are randomly selected from Literature [4]; 15 samples will be selected to learn and train the grey neural network model (GMBPNN) and the remaining 6 samples will serve as the verification samples. The training sample set and verification sample set are shown in Table 1 and Table 2 . Where, $c$ is the friction force of soil layer $(\mathrm{kPa}) ; \varphi$ is the internal friction angle $\left(^{\circ}\right) ; H$ is the burial depth of tunnel (m); $D$ is the diameter of tunnel (m); $n$ is the consolidation degree of soil (\%); Es is the elastic modulus of secant line (MPa); $p$ is the grouting pressure value (MPa); $F$ is the thrust pressure of shield machine (MN); $v$ is the advance rate of shield machine (mm.min-1).

Table 1 Training Sample Set of Landslide

\begin{tabular}{|c|c|c|c|c|c|c|c|c|c|}
\hline No. & $\varphi /\left(^{\circ}\right)$ & $H / \mathrm{m}$ & $D / \mathrm{m}$ & $n / \%$ & $C / \mathrm{kPa}$ & $\begin{array}{c}E s / M P \\
a\end{array}$ & $\begin{array}{c}p / \mathrm{MP} \\
\mathrm{a}\end{array}$ & $\begin{array}{c}F / M \\
\mathrm{~N}\end{array}$ & $V /\left(\mathrm{mm} \cdot \mathrm{min}^{-1}\right)$ \\
\hline 1 & 16.4 & 8.4 & 6.34 & 1.7 & 11.7 & 6.48 & 0.3 & 14 & 30 \\
\hline 2 & 19.5 & 6.1 & 6.34 & 1.4 & 11.2 & 8.26 & 0.3 & 14 & 40 \\
\hline 3 & 14.7 & 10.4 & 6.34 & 1.8 & 11.8 & 5.67 & 0.4 & 14 & 20 \\
\hline 4 & 14.1 & 11.4 & 6.34 & 1.8 & 11.9 & 5.35 & 0.4 & 14 & 20 \\
\hline 5 & 13.4 & 12.7 & 6.34 & 2 & 12 & 5.01 & 0.4 & 14 & 20 \\
\hline 6 & 23.9 & 12 & 6.4 & 1.5 & 15.3 & 3.87 & 0.3 & 31.65 & 40 \\
\hline 7 & 20.7 & 13.8 & 6.4 & 1.7 & 36.7 & 7.26 & 0.25 & 31.65 & 60 \\
\hline 8 & 12.4 & 14.2 & 6.4 & 1.7 & 32.4 & 11.17 & 0.25 & 31.65 & 60 \\
\hline 9 & 30 & 14.5 & 6.4 & 1.5 & 43.6 & 9.12 & 0.25 & 31.65 & 60 \\
\hline 10 & 9.2 & 14.5 & 6.4 & 1.7 & 32.2 & 7.26 & 0.25 & 31.65 & 60 \\
\hline 11 & 14.5 & 14.5 & 6.4 & 1.7 & 34.2 & 7.26 & 0.25 & 31.65 & 50 \\
\hline 12 & 44.9 & 12 & 6.25 & 0.9 & 234 & 35.02 & 0.2 & 33 & 30 \\
\hline 13 & 30.3 & 24 & 6.25 & 1.2 & 680 & 23.57 & 0.3 & 33 & 30 \\
\hline 14 & 29.8 & 23 & 6.25 & 1.1 & 650 & 21.83 & 0.2 & 33 & 40 \\
\hline 15 & 14.4 & 10.9 & 6.34 & 2 & 11.8 & 5.53 & 0.4 & 14 & 20 \\
\hline
\end{tabular}

Table2 Validation Sample Set

\begin{tabular}{|c|c|c|c|c|c|c|c|c|c|}
\hline No. & $\varphi /\left(^{\circ}\right)$ & $H / \mathrm{m}$ & $D / \mathrm{m}$ & $n / \%$ & $C / \mathrm{kPa}$ & $\begin{array}{c}E s / M P \\
\text { a }\end{array}$ & $\begin{array}{c}p / \mathrm{MP} \\
\mathrm{a}\end{array}$ & $\begin{array}{c}F / M \\
\mathrm{~N}\end{array}$ & $V /\left(\mathrm{mm} \cdot \mathrm{min}^{-1}\right)$ \\
\hline 1 & 4.77 & 5.91 & 9.78 & 0.35 & 11.8 & 15.2 & 6.34 & 1.7 & 14 \\
\hline 2 & 9.71 & 5.42 & 13.3 & 0.25 & 28.1 & 12.8 & 6.4 & 1.5 & 31.65 \\
\hline 3 & 5.37 & 11.17 & 14.5 & 0.25 & 32.4 & 10.7 & 6.4 & 1.7 & 31.65 \\
\hline 4 & 9.77 & 40.75 & 20 & 0.3 & 310 & 44.1 & 6.25 & 1.3 & 33 \\
\hline \multirow[t]{2}{*}{5} & 7.25 & 5.22 & 11.9 & 0.4 & 11.9 & 13.8 & 6.34 & 2 & 14 \\
\hline & 7.46 & 5.21 & 12 & 0.25 & 12.1 & 13.7 & 6.34 & 1.7 & 31.65 \\
\hline
\end{tabular}

\section{Data standardization processing}

During learning process of BP neural network, the inconsistency of dimension can cause slow learning speed and affect the prediction precision; to improve the learning speed and prediction precision, the standardization processing shall be conducted for training sample set and validation sample set and the standardization processing for data set shall be conducted by Equation (16) according to the features for data set of landslide.

$$
\widehat{x_{i}}=\frac{x_{i}-\min (x)}{\max (x)-\min (x)}
$$

Where, $\widehat{x}_{i}$ is the value of landslide index after standardization processing; $x_{i}$ is the original data of landslide index; $\max (x)$ and $\min (x)$ are respectively the maximum value and minimum value in index data of landslide.

\section{Results and analysis}

The training sample set of landslide in Table 1 can be used as the learning sample of model to train the model and the verification sample set in Table 2 can be used to train the model; meanwhile, to verify the effectiveness and efficiency of model put forth in the Thesis, the single grey model and 
BP neural network model serve as the contrast models, the prediction values and measured values of the models are shown in Fig. 2 and the verification error is shown in Table 3.

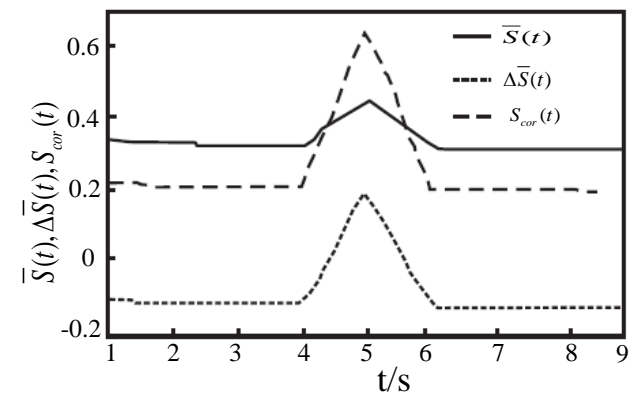

Fig.2 Comparison Between Measured Value and Prediction Value of Landslide Table 3 Error of Verification Sample

\begin{tabular}{cccc}
\hline No. & Grey model & BPMNN & My Method \\
\hline 1 & 10.87 & -4.24 & 1.23 \\
2 & -7.24 & -6.76 & 1.51 \\
3 & -9.02 & -4.13 & -0.98 \\
4 & 4.7 & -0.77 & -1.92 \\
5 & 7 & -4.03 & -0.8 \\
6 & 6.2 & 3.02 & 1.01 \\
\hline
\end{tabular}

Fig. 2 indicates that the difference between prediction result and measured value of grey model is relatively large, while the prediction result of BP neural network model is more accurate than that of grey model because the non-linear law of landslide is hard to be accurately simulated by grey model, but the non-linear function can be approached more accurately by BP neural network model. The difference between prediction value and measured value of combined model is the least, while the precision is the highest; the error is less than that of single grey model and BP neural network model and the precision is higher, so it is indicated that the combined model can sufficiently make full use of the information of original data to make the insufficient information obtained by single model and the advantages of grey model and BP neural network model can be fully utilized to effectively improve the prediction precision.

\section{Conclusions}

To improve the prediction precision of landslide, a prediction method of landslide by data mining technology is put forth. First, the grey model is used to predict the law of landslide in the method, and then the non-linear mapping relation between landslide and random variable is established through BP neural network to obtain the final prediction value of landslide. The verification test indicates that by comparison to single model, the shortcomings of single mode are overcome in the Thesis and the prediction precision of landslide is improved.

\section{Acknowledgement}

The National Natural Science Foundation of China under Grant No.51379201; The National Natural Science Foundation of China under Grant No.51404239.

\section{Reference}

[1] Yu D. Intuitionistic fuzzy theory based typhoon disaster evaluation in Zhejiang Province, China: a comparative perspective[J]. Natural Hazards, 2015, 75(3):2559-2576.

[2] Xiong X, Lu Y, Muhammad N. A New Method of Multi-criteria Group Decision Making Based on Hesitant-valued Intuitionistic Fuzzy and Osculating Value[C]// International Conference on Logistics, Engineering, Management and Computer Science (LEMCS 2015). Atlantis Press, 2015.

[3] Liu C, Li W, Lu P, et al. Susceptibility Evaluation and Mapping of CHINA'S Landslide 
Disaster Based on Multi-Temporal Ground and Remote Sensing Satellite Data[J]. ISPRS International Archives of the Photogrammetry, 2012, XXXIX-B8:45-50.

[4] Yang F, Yuan Q, Du S, et al. Reserving relief supplies for earthquake: a multi-attribute decision making of China Red Cross[J]. Annals of Operations Research, 2014:1-27. 